EPJ Web of Conferences 49, 18002 (2013)

DOI: $10.1051 /$ epjconf/20134918002

(C) Owned by the authors, published by EDP Sciences, 2013

\title{
Lepton number violation at the LHC with leptoquarks and diquarks ${ }^{\star}$
}

\author{
Hiroaki Sugiyama ${ }^{1}$ \\ ${ }^{1}$ Department of Physics, University of Toyama, Toyama 930-8555, Japan
}

\begin{abstract}
We investigate a model in which tiny neutrino masses are generated at the two-loop level by using scalar leptoquark and diquark multiplets. The diquark can be singly produced at the LHC, and it can decay into a pair of leptoquarks through the lepton number violating interaction. Subsequent decays of the two leptoquarks can provide a clear signature of the lepton number violation, namely two QCD jets and a pair of same-signed charged leptons without missing energy. We show that the signal process is not suppressed while neutrino masses are appropriately suppressed.
\end{abstract}

\section{Introduction}

Although the lepton number is conserved in the standard model (SM) of particle physics, the addition of the Majorana mass term of neutrinos $\left(\bar{v} v^{c}\right.$ where $c$ denotes the charge conjugation) breaks the lepton number conservation by two units. The measurement of the lepton number violating $(\mathrm{L \# V)}$ processes such as the neutrinoless double beta decay is extremely important because it gives evidence that neutrinos are Majorana particles. Such processes are naively expected to be very rare because neutrino masses are very small. However, in models of radiatively generated neutrino masses, a trilinear coupling constant for light (e.g. TeV-scale) scalars can be more fundamental than light neutrino masses as the $\mathrm{L \# V}$ parameter at the accessible energy scale. Then, $\mathrm{L \# V}$ processes via the trilinear coupling constant can be significant at the TeVscale even if the neutrino masses are suppressed enough.

New particles related to the neutrino mass generation are usually produced via the electroweak interaction, and therefore the production cross sections are not so significant at the LHC. However, new particles in the loop of the radiative seesaw models can be charged under the $\mathrm{SU}(3)_{C}[2-4]$. Such a colored particle can easily be produced at hadron colliders. In this presentation, we investigate a radiative seesaw model with a scalar leptoquark multiplet and a scalar diquark multiplet.

\section{The Model}

Scalar particles listed in Table 1 are introduced to the SM. The model is briefly mentioned in Ref. [2]. The model includes a scalar leptoquark multiplet $S_{\mathrm{LQ}}$ whose lepton number $(L \#)$ and baryon number $(B \#)$ are 1 and $1 / 3$, respectively. Under the SM gauge group, the $S_{\mathrm{LQ}}$ is assigned to the same representation of right-handed downtype quarks; a $\underline{\mathbf{3}}$ representation of $\mathrm{SU}(3)_{C}$, a singlet under

${ }^{\star}$ This presentation is based on our work in Ref. [1].
Table 1. List of scalar particles added to the SM.

\begin{tabular}{c||c|c|c||c|c} 
& $\mathrm{SU}(3)_{C}$ & $\mathrm{SU}(2)_{L}$ & $\mathrm{U}(1)_{Y}$ & $L \#$ & $B \#$ \\
\hline \hline$S_{\mathrm{LQ}}^{\alpha}$ & $\underline{\mathbf{3}}$ & $\underline{\mathbf{1}}$ & $-1 / 3$ & 1 & $1 / 3$ \\
\hline$S_{\mathrm{DQ}}^{\alpha \beta}$ & $\underline{\mathbf{6}}$ & $\underline{\mathbf{1}}$ & $-2 / 3$ & 0 & $2 / 3$
\end{tabular}

$\mathrm{SU}(2)_{L}$, and hypercharge $Y=-1 / 3$. We also introduce a scalar diquark multiplet $S_{\mathrm{DQ}}$ whose baryon number is $2 / 3$. We take $S_{\mathrm{DQ}}$ as a $\underline{\mathbf{6}}$ representation of $\mathrm{SU}(3)_{C}$, a singlet under SU(2) $)_{L}$, and a $Y=-2 / 3$ field. Note that $S_{\mathrm{DQ}}^{\alpha \beta}=S_{\mathrm{DQ}}^{\beta \alpha}$, where $\alpha$ and $\beta$ (=r,g,b) denote the color indices.

The baryon number conservation is imposed to the model such that the proton decay is forbidden. We introduce the soft-breaking term (see the next paragraph) of the lepton number conservation to the scalar potential in order to generate Majorana neutrino masses. The Yukawa interactions with the leptoquark and diquark are given by

$$
\begin{aligned}
\mathcal{L}_{\text {Yukawa }}= & -\left\{\overline{L_{\ell}^{c}}\left(Y_{L}\right)_{\ell i} i \sigma_{2} Q_{i}^{\alpha}+\overline{\left(\ell_{R}\right)^{c}}\left(Y_{R}\right)_{\ell i} u_{i R}^{\alpha}\right\}\left(S_{\mathrm{LQ}}^{\alpha}\right)^{*} \\
& -\overline{\left(d_{i R}^{\alpha}\right)^{c}}\left(Y_{s}\right)_{i j} d_{j R}^{\beta}\left(S_{\mathrm{DQ}}^{\alpha \beta}\right)^{*}+\text { H.c. },
\end{aligned}
$$

where $\sigma_{a}(a=1-3)$ are the Pauli matrices. We choose the diagonal bases of mass matrices for the charged leptons and down-type quarks. Then, the $\mathrm{SU}(2)_{L}$ partner of $d_{i L}$ is described as $u_{i L}^{\prime}=\left(V_{\mathrm{CKM}}^{\dagger}\right)_{i j} u_{j L}$, where $V_{\mathrm{CKM}}$ is the Cabibbo-Kobayashi-Maskawa (CKM) matrix and $u_{j}=\left(u_{j R}, u_{j L}\right)^{T}$ are mass eigenstates of up-type quarks. Mass eigenstates $v_{i L}$ of neutrinos are given by $v_{i L}=\left(U_{\text {MNS }}^{\dagger}\right)_{i \ell} v_{\ell L}$, where $U_{\text {MNS }}$ is the Maki-NakagawaSakata (MNS) matrix. The Yukawa matrices $\left(Y_{L}, Y_{R}\right.$, and $\left.Y_{S}\right)$ are $3 \times 3$ matrices under the lepton flavor $(\ell=e, \mu, \tau)$ and the down-type quark flavor $(i j=1-3)$. While $Y_{L}$ and $Y_{R}$ are general complex matrices, $Y_{s}$ is a symmetric matrix $\left(Y_{s}^{T}=Y_{s}\right)$. Note that neutrinos interact with the leptoquark only through $Y_{L}$. 


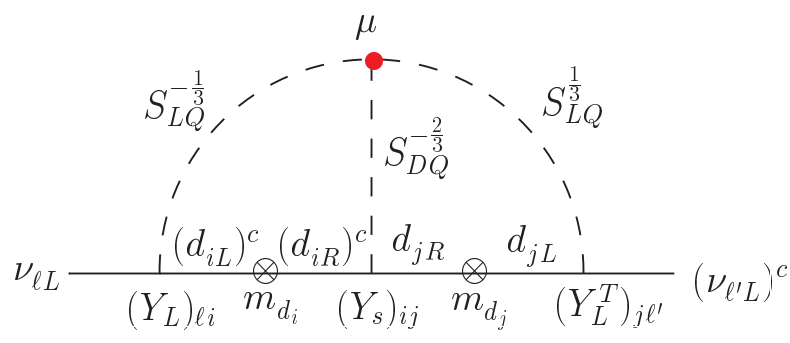

Figure 1. The two-loop diagram for the neutrino mass generation in the model.

In the scalar potential of the model, we introduce the following three-point interaction:

$$
\mu\left(S_{\mathrm{LQ}}^{\alpha}\right)^{*}\left(S_{\mathrm{LQ}}^{\beta}\right)^{*} S_{\mathrm{DQ}}^{\alpha \beta}+\text { H.c. }
$$

The coupling constant $\mu$ softly breaks the lepton number conservation by two units while the baryon number is conserved. There is no other possible soft-breaking term of the lepton number and/or the baryon number.

The neutrino mass term $\frac{1}{2}\left(M_{v}\right)_{\ell \ell^{\prime}} \overline{v_{\ell L}}\left(v_{\ell^{\prime} L}\right)^{c}$ in the flavor basis is generated by a two-loop diagram in Fig. 1 including the leptoquark and the diquark. The diagram is similar to the one in the Zee-Babu model (ZBM) [6] although $\mathrm{SU}(3)_{C}$-singlet particles in the loop are replaced with colored particles. Thus, we refer to this model as the colored Zee-Babu model (cZBM). The neutrino mass matrix in the cZBM is calculated as

$$
\left(M_{v}\right)_{\ell \ell^{\prime}}=+24 \mu\left(Y_{L}^{*}\right)_{\ell i} m_{d_{i}}\left(Y_{s}\right)_{i j} I_{i j} m_{d_{j}}\left(Y_{L}^{\dagger}\right)_{j \ell^{\prime}},
$$

where $I_{i j}$ is the two loop function (See Ref. [5] for the explicit form of $I_{i j}$ ).

Hereafter, we restrict ourselves to the simplest scenario where $Y_{R}$ (which does not contribute to $\left(M_{v}\right)_{\ell \ell^{\prime}}$ ) is small enough to be ignored. A benchmark point in the parameter space of the cZBM is shown in Appendix A.

\section{New colored scalars at the LHC}

\subsection{Leptoquark}

The leptoquarks have been searched at the Tevatron and the LHC. The most stringent lower bound on $m_{\mathrm{LQ}}$ at $95 \%$ confidence level is set as $830 \mathrm{GeV}(840 \mathrm{GeV})$ by the recent CMS result at $\sqrt{s}=7 \mathrm{TeV}$ with $5.0 \mathrm{fb}^{-1}$ integrated luminosity [7] (See also Refs. [8]).

The leptoquark induces various LFV processes. At the tree level, four-fermion operators (two left-handed leptons and two left-handed quarks) are generated by integrating leptoquarks out. Operators $\left(\overline{e_{L}} \gamma^{\mu} \mu_{L}\right)\left(\overline{u_{L}} \gamma_{\mu} u_{L}\right)$ and $\left(\overline{v_{\ell L}} \gamma^{\mu} v_{\ell^{\prime} L}\right)\left(\overline{d_{L}} \gamma_{\mu} s_{L}\right)$ are strongly constrained by the $\mu-e$ conversion search and the $K$ meson decay measurement, respectively [9]. For the benchmark point in Appendix A, we have $\left|\left(Y_{L}\right)_{e 1}\left(Y_{L}^{*}\right)_{\mu 1}\right| /\left(4 \sqrt{2} G_{F} m_{\mathrm{LQ}}^{2}\right)=6.1 \times 10^{-11}$ and $\left|\left(Y_{L}\right)_{\ell 1}\left(Y_{L}^{*}\right)_{\ell^{\prime} 2}\right| /\left(4 \sqrt{2} G_{F} m_{\mathrm{LQ}}^{2}\right) \lesssim 10^{-7}$ which satisfy constraints shown in Ref. [9], where $G_{F}=1.17 \times 10^{-5} \mathrm{GeV}^{-2}$.
At the loop level, effects of leptoquarks on charged lepton transitions, i.e., $\ell_{i} \rightarrow \ell_{j} \gamma$, have also been studied [10]. Since we assume that $S_{\mathrm{LQ}}$ has the Yukawa interaction only with the left-handed quarks (namely $Y_{R}=0$ ), the contribution from the top quark loop does not give a large enhancement of $\ell_{i} \rightarrow \ell_{j} \gamma$. Then, the branching ratio of $\mu \rightarrow e \gamma$ is calculated as $\operatorname{BR}(\mu \rightarrow e \gamma)=$ $3 \alpha_{\mathrm{EM}}\left|\left(Y_{L} Y_{L}^{\dagger}\right)_{e \mu}\right|^{2} /\left(256 \pi G_{F}^{2} m_{\mathrm{LQ}}^{4}\right)$, where $\alpha_{\mathrm{EM}}=1 / 137$. For example, a benchmark point shown in Appendix A gives $\operatorname{BR}(\mu \rightarrow e \gamma)=6.5 \times 10^{-13}$ which satisfies the current upper bound $\left(2.4 \times 10^{-12}\right.$ at $90 \%$ confidence level $)$ in the MEG experiment [11].

\subsection{Diquark}

At the LHC, the diquark $S_{\mathrm{DQ}}$ in the cZBM would be singly produced by the annihilation of two down-type quarks via $\left(Y_{s}\right)_{11}$. The $\left(Y_{s}\right)_{11}$ in the cZBM is less constrained by the neutrino oscillation data because its contribution to neutrino masses is suppressed by $m_{d}^{2} / m_{\mathrm{DQ}}^{2}$. If we assume $\left(Y_{s}\right)_{11}=0.1$ and $m_{\mathrm{DQ}}=4 \mathrm{TeV}$, the single production cross section $\sigma\left(d d \rightarrow S_{\mathrm{DQ}}\right)$ is about $5 \mathrm{fb}$ at the LHC with $\sqrt{s}=14 \mathrm{TeV}$ [12]. Note that the CMS experiment at $\sqrt{s}=7 \mathrm{TeV}$ with $1 \mathrm{fb}^{-1}$ integrated luminosity excludes diquark masses between $1 \mathrm{TeV}$ and $3.52 \mathrm{TeV}$ at $95 \%$ confidence level by assuming the diquark decay into two QCD jets for the $E_{6}$ diquark which couples with an up-type quark and a down-type quark [13].

The diquark induces flavor changing neutral current processes in the down-type quark sector. Especially, it gives tree-level contributions to $K^{0}-\overline{K^{0}}, B_{d}^{0}-\overline{B_{d}^{0}}$ and $B_{s}^{0}-\overline{B_{s}^{0}}$ mixings, resulting in strong constraints on $Y_{s}$. By using the notations in Ref. [14], the benchmark point in Eqs. (4) gives $\widetilde{C}_{K}^{1}=-\left(Y_{s}^{*}\right)_{11}\left(Y_{s}\right)_{22} /\left(2 m_{\mathrm{DQ}}^{2}\right)=0$. Similarly, we have $\widetilde{C}_{B_{d}}^{1}=+1.2 \times 10^{-12} \mathrm{GeV}^{-2}$ and $\widetilde{C}_{B_{s}}^{1}=0$. These values satisfy the constraints obtained in Ref. [14].

The diquark in the cZBM decays into not only a pair of the down-type quarks but also a pair of leptoquarks. Subsequently, $50 \%$ of each leptoquark decays into an up-type quark (a down-type quark) and a charged lepton (a neutrino). Then, the model provides a characteristic signature in Fig. 2, whose final state consists of two QCD jets and the same-signed charged lepton pair without missing energy. The decay chain of the diquark can be fully reconstructed at the LHC. This signature can be a smoking gun for the lepton number violation because no lepton number is taken away by invisible particles. There is no irreducible background in principle because the SM conserves the lepton number. It would be also very rare that the SM process mimics the signal process because the leptons in the signal events are too energetic to be produced in the SM process.

It should be emphasized that the event rate of the process is not necessarily suppressed though the full process picks up all new coupling constants relevant to the small neutrino masses (namely, $Y_{s}, \mu$, and $Y_{L}$ ). One reason for that is because the process in Fig. 2 does not have suppressions with the two-loop factor $1 /\left(16 \pi^{2}\right)^{2}$ and with down-type quark masses, which are used for tiny neu- 


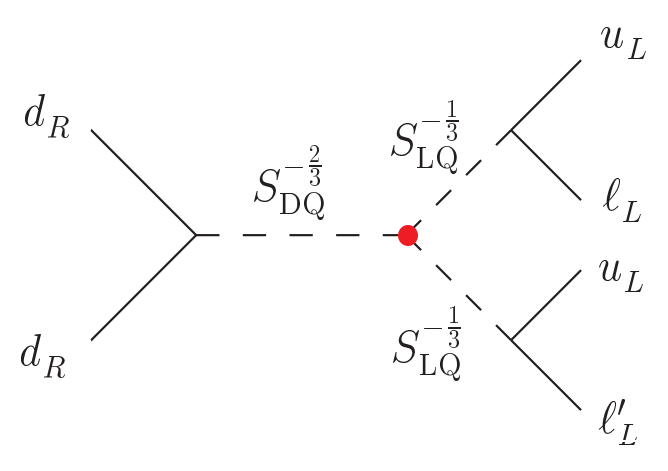

Figure 2. The same-signed charged lepton signature without missing energy at the LHC.

trino masses. The other reason is that on-shell productions of a diquark and leptoquarks are utilized as $\sigma(d d \rightarrow$ $\left.S_{\mathrm{DQ}}\right) \mathrm{BR}\left(S_{\mathrm{DQ}} \rightarrow S_{\mathrm{LQ}} S_{\mathrm{LQ}}\right)\left[\sum_{\ell, i} \mathrm{BR}\left(S_{\mathrm{LQ}} \rightarrow \ell_{L} u_{i L}\right)\right]^{2}$; even if a partial decay width is controlled by a small coupling constant (e.g., $S_{\mathrm{LQ}} \rightarrow \ell_{L} u_{i L}$ via $\left.\left(Y_{L}\right)_{\ell i}\right)$, its branching ratio becomes sizable when the total decay width is also controlled by small coupling constants. In this scenario, the cZBM seems the new physics model which is the most easily probed at the LHC and takes us to the top of the energy frontier.

For the benchmark point shown in the Appendix A, the decay branching ration for $S_{\mathrm{DQ}} \rightarrow S_{\mathrm{LQ}} S_{\mathrm{LQ}}$ is $85 \%$. Then, $15 \%(7 \%)$ of $S_{\mathrm{LQ}}$ decays into a charm quark (a top quark) associated with an electron or a muon. Decays into an up quark are negligible for the bench mark point. The decay into a tau lepton might not be reliable because it gives missing neutrinos. Even if $S_{\mathrm{LQ}}$ decays into a top quark, hadronic decays $(68 \%)$ of $W^{ \pm}$from the top quark decay have no missing energy. As a result, the cross section for L\#V events without missing energy is about $0.18 \mathrm{fb}$ at the LHC with $\sqrt{s}=14 \mathrm{TeV}$ for the benchmark point.

\section{Conclusions}

We have studied a model for neutrino mass generation with the scalar leptoquark $S_{\mathrm{LQ}}$ and diquark $S_{\mathrm{DQ}}$. Tiny Majorana neutrino masses are induced at the two-loop level where the colored particles are involved in the loop.

The model gives a distinctive signature at the LHC, namely $p p \rightarrow S_{\mathrm{DQ}} \rightarrow S_{\mathrm{LQ}} S_{\mathrm{LQ}} \rightarrow \ell^{-} \ell^{\prime-} j j$ without missing energy, which would be a clear evidence of the lepton number violation. We have shown that the lepton number violating process is not suppressed in this model while Majorana neutrino masses are highly suppressed.

\section{A A benchmark point}

Here, we show a benchmark point of the model:

$$
\begin{aligned}
& Y_{L}=\left(\begin{array}{rcc}
8.1 \times 10^{-5} & 4.0 \times 10^{-2} & -7.0 \times 10^{-3} \\
-4.9 \times 10^{-5} & 5.3 \times 10^{-2} & 4.4 \times 10^{-2} \\
3.1 \times 10^{-5} & -2.3 \times 10^{-2} & 8.9 \times 10^{-2}
\end{array}\right), \\
& Y_{s}=\left(\begin{array}{ccc}
1.0 \times 10^{-1} & 0 & 0 \\
0 & 0 & -1.2 \times 10^{-2} \\
0 & -1.2 \times 10^{-2} & -3.8 \times 10^{-4}
\end{array}\right), \\
& \mu=1 \mathrm{TeV}, \quad m_{\mathrm{LQ}}=1 \mathrm{TeV}, \quad m_{\mathrm{DQ}}=4 \mathrm{TeV} .
\end{aligned}
$$

The benchmark point gives the following values: $\sin ^{2} 2 \theta_{23}=1, \sin ^{2} 2 \theta_{13}=0.1, \sin ^{2} 2 \theta_{12}=0.87, \delta=0$, $m_{1} \simeq 0, \Delta m_{31}^{2}=2.4 \times 10^{-3} \mathrm{eV}^{2}>0$, and $\Delta m_{21}^{2}=$ $7.6 \times 10^{-5} \mathrm{eV}^{2}$. The effective mass relevant for the neutrinoless double beta decay is $\left(M_{v}\right)_{e e} \simeq 1.5 \times 10^{-3} \mathrm{eV}$.

\section{References}

[1] M. Kohda, H. Sugiyama and K. Tsumura, Phys. Lett. B 718, 1436 (2013).

[2] K. S. Babu and C. N. Leung, Nucl. Phys. B 619, 667 (2001).

[3] D. Aristizabal Sierra, M. Hirsch and S. G. Kovalenko, Phys. Rev. D 77, 055011 (2008).

[4] P. Fileviez Perez and M. B. Wise, Phys. Rev. D 80, 053006 (2009).

[5] K. S. Babu and C. Macesanu, Phys. Rev. D 67, 073010 (2003).

[6] A. Zee, Nucl. Phys. B 264, 99 (1986); K. S. Babu, Phys. Lett. B 203, 132 (1988).

[7] S. Chatrchyan et al. [CMS Collaboration], Phys. Rev. D 86, 052013 (2012).

[8] G. Aad et al. [ATLAS Collaboration], Phys. Lett. B 709, 158 (2012) [Erratum-ibid. 711, 442 (2012)]; Eur. Phys. J. C 72, 2151 (2012); S. Chatrchyan et al. [CMS Collaboration], JHEP 1212, 055 (2012).

[9] M. Carpentier and S. Davidson, Eur. Phys. J. C 70, 1071 (2010).

[10] R. Benbrik and C. -K. Chua, Phys. Rev. D 78, 075025 (2008).

[11] J. Adam et al. [MEG Collaboration], Phys. Rev. Lett. 107, 171801 (2011).

[12] O. Cakir and M. Sahin, Phys. Rev. D 72, 115011 (2005); T. Han, I. Lewis and Z. Liu, JHEP 1012, 085 (2010).

[13] S. Chatrchyan et al. [CMS Collaboration], Phys. Lett. B 704, 123 (2011).

[14] M. Bona et al. [UTfit Collaboration], JHEP 0803, 049 (2008). 